\title{
Long-term superiority of CABG surgery for three-vessel disease confirmed
}

The 5-year outcomes of the SYNTAX trial confirm that CABG surgery remains a superior revascularization strategy to percutaneous coronary intervention (PCI) in the prespecified subgroup of patients with three-vessel disease, particularly those with diabetes mellitus or a high SYNTAX score (indicating complex lesions). However, these results should be considered observational and hypothesisgenerating, because the prerequisite of noninferiority of the primary end point was not met in the overall study population.

Of the 1,800 patients included in the SYNTAX trial, 1,095 had three-vessel disease. In this subgroup, 549 were randomly allocated to undergo CABG surgery, whereas 546 received PCI with a first-generation paclitaxel-eluting stent. No differences existed in either the extent or the complexity of coronary artery disease between the two groups. Patients assigned to CABG surgery had longer waiting times, procedural duration, and in-hospital stay after surgery than those undergoing PCI.

At 5 -year follow-up, $88 \%$ and $96 \%$ of the patients with three-vessel disease randomly allocated to CABG surgery or PCI, respectively, were available for analysis. Major adverse cardiac and cerebrovascular events (MACCE; a composite of all-cause death, stroke, myocardial infarction, and repeat revascularization) occurred significantly more frequently with PCI than with CABG surgery (37.5\% vs $24.2 \%$; HR 1.70, 95\% CI 1.36-2.13, $P<0.001)$. The composite safety end point (death, stroke, and myocardial infarction) was also more commonly observed after PCI than after CABG surgery (22.0\% vs $14.0 \%$; HR 1.64, 95\% CI 1.22-2.20, $P<0.001)$. This difference was driven by increased rates of all-cause death and myocardial infarction with PCI. No significant difference in the incidence of stroke was reported between the two groups. However, repeat revascularization was necessary significantly more frequently after PCI than after CABG surgery (25.4\% vs $12.6 \%$; HR 2.24, 95\% CI 1.65-3.04, $P<0.001)$. PCI was a significant independent predictor of the composite safety end point (HR 1.81) and of all-cause death (HR 1.81).

In patients with a low SYNTAX score (0-22), neither the rate of MACCE nor that of the composite safety end point significantly differed between the two revascularization strategies. However, PCI was associated with an increased need for repeat revascularization. In patients with intermediate or high lesion complexity (SYNTAX score $\geq 23$ ), rates of MACCE and the composite safety end point were significantly lower with CABG surgery than with PCI. Among the patients with three-vessel disease in the SYNTAX trial, 296 had medically treated diabetes. The rate of MACCE in these patients was significantly higher in the 153 who received PCI than in the 143 who underwent CABG surgery (45.5\% vs $23.6 \%$; HR 2.30, $95 \%$ CI $1.50-3.55, P<0.001)$.

Overall, these 5-year data confirm the guideline-recommended preference for CABG surgery over PCI for patients with three-vessel disease, especially those with complex lesions or diabetes. However, the evolution of PCI technology from balloon angioplasty and bare-metal stents to drug-eluting stents and biodegradable scaffolds has improved outcomes with this strategy. Similarly, technical advances in CABG surgery have enhanced postoperative outcomes. Therefore, the SYNTAX investigators call for "randomized trials ... to assess revascularization options in the era of newer-generation drugeluting stents". Furthermore, awareness of functional (rather than purely anatomical) assessment of coronary lesions in rising. Functional impairment can be measured by fractional flow reserve (FFR). "The impact of FFR-guided revascularization on the PCI vs CABG [surgery] treatment effect," say the investigators, "will be determined in the FAME III trial.”

Gregory B. Lim

Original article Head, S. J. et al. Coronary artery bypass grafting vs. percutaneous coronary intervention for patients with three-vessel disease: final five-year follow-up of the SYNTAX trial. Eur. Heart J. doi:10.1093/eurheartj/ehu213

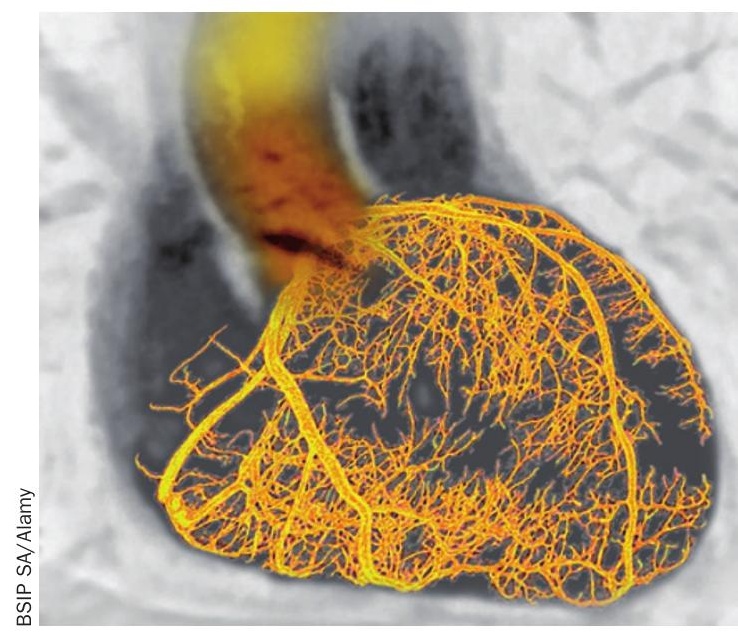

